\title{
14
}

\section{Panel Description: The future of business meetings - possibilities and problems of computer support in virtual organisations}

\author{
Panel Chair: \\ J. M. Haake, GMD-IPSI \\ Panel Participants: \\ M. Bever, IBM-ENC \\ J. Bumiller, Daimler-Benz Research \\ D. Martin, SMART Technologies
}

Due to globalisation of markets, business processes of today are characterized by a change from individual work to team work as well as by a shift from single, independently acting corporations to virtual organisations operating as dynamic networks. On the other hand, trends emerging in this context are joint ventures and outsourcing - trends which result in an increased complexity and frequency of communication, co-ordination and co-operation within companies as well as across organisational boundaries. As a consequence, meetings at all levels have become more frequently over the past decade. This development calls for improved and enhanced communication facilities at an internal level, e.g., between various teams of a company, and at an external level, e.g., between a corporation and its suppliers or customers.

So far, efforts to electronically support synchronous communication (e.g., meetings by video-conferencing systems) have mostly been limited in terms of integration. The main reasons for insufficient progress - or even failure - of most current meeting support systems are their lack of flexibility and their insufficient integration with asynchronous modes of co-operation and related applications (e.g., workflow management systems, multimedia collaboration teleservices). Problems arising from this deficit are manyfold: Documents of a meeting participant are unusable since their format is incompatible to the electronic meeting support system; data are unaccessable due to missing interconnectivity; particular expertise required during a session is not available since there is no support for immediate electronic consultation of absent group members or external experts - or even more important, the only person who is authorised to make a crucial decision at some point of a negotiation can not be consulted.

Such deficits lead to incompensatory delays, decrease "speed to market" or reduce the quality of strategic managerial decisions. Moreover, they inevitably cause considerable costs for the intense communication and cooperation activities typically required in virtual organisations. 
This financial overhead could be drastically reduced if integrated, flexible meeting support systems based on powerful telecommunication services and communication channels were in place which accounted for the particular requirements of virtual organisations.

There are a number of technologies available for implementing meeting support, such as: the integration of the computer and the phone, radio-linked notepads, desktop teleconferencing, video conferencing rooms, distributed coupled meeting rooms, etc.

The integration of the PC (and computers in general) with the telephone is what is driving a lot of the possibilities for having virtual meetings with our co-workers. Up to now, the ubiqitous availability of the telephone, the fact that everyone knows how to make a telephone work, and the inexpensive cost of acquisition and operation has focused electronic meetings through the telephone network.

But as the cost of being connected via video, audio, and data conferencing technologies comes down dramatically, as the 55 million people that buy new PC's this year get more familiar with the inherent technologies, and bandwidth becomes cheaper and more availability, there is likely to be a wide-spread adoption in the use of computer based telecommunications for the sharing of information.

There is likely to be some significant variation in how well organizations do in adopting and using this technology based upon the types of meetings that they conduct, the availability of cross-platform solutions, the ease of use of these technologies, the bridging of same time data access to different time information tools, the provision of means for integrating information, etc. Similarily, different kinds of meetings and situations may require different support thus leading to different kinds of meeting support (each with the potential of using different technology).

All of the problems are not solved, but the impetus is there for a whole new generation of tools to be accepted by workers in the world community. We have solved significant problems in getting people to accept new ways of conducting meetings at a distance and in the same meeting room. This will result in broader acceptance of the enabling software technologies that provide for more productive meetings in the coming months and years.

The goal of this panel was to discuss the requirements, potential solutions and problems of meeting support in virtual organisations.

\section{THE PANELISTS}

Joerg M. Haake is a department manager at the Integrated Information and Publication Systems Institute of GMD - German National Research Center for Information Technology where he is leading research in hypermedia-based collaboration support systems.

Dr. Martin Bever is manager of Telecooperation IBM Deutschland Informationssysteme $\mathrm{GmbH}$. He is managing a research department at the IBM European Networking Center. His main research interest is in distributed telecooperation applications.

Dr. Johannes Bumiller is a rearcher at Daimler-Benz Research Information Technology. His main interests are in Computer Supported Cooperative Work.

David Martin, President of SMART Technologies Inc. of Calgary, Alberta, Canada. Since 1987 he has led the Company to develop hardware and software products that allow the interconnection of people in electronic meetings. The Company is at the forefront of the integration of computers with the telephone through its alliance with Intel Corporation. 